\title{
WHEN DOES THE ZERO-ONE LAW HOLD?
}

\author{
TOMASZ LUCZAK AND JOEL SPENCER
}

\section{INTRODUCTION}

In 1960 Paul Erdös and Alfred Rényi [ER] began the subject of random graphs. The 1985 book of Béla Bollobás [B] provides the standard reference for this field. In modern terminology the random graph $G(n, p)$ is a graph on vertex set $[n]=\{1, \ldots, n\}$ where each pair $i, j$ of vertices are adjacent with independent probability $p$. More accurately, $G(n, p)$ is a probability space over the space of graphs on vertex set $[n]$. For any property $A$ of graphs there is a probability, denoted $\operatorname{Pr}[G(n, p) \models A]$, that $G(n, p)$ satisfies $A$.

In their very title, "On the evolution of random graphs," Erdös and Rényi envisioned a dynamic process, $G(n, p)$ changing character as $p$ moved from zero to one. They discovered (as did their many successors) that for many natural properties $A \operatorname{Pr}[G(n, p) \vDash A]$ was usually near zero or near one and made the jump from near zero to near one (or back again) in a very narrow range. The placement of this critical range of $p$ depended on $n$. For example, let $A$ be the property of containing a triangle. There are $\left(\begin{array}{l}n \\ 3\end{array}\right) \sim n^{3} / 6$ potential triangles, each is a triangle in $G(n, p)$ with probability $p^{3}$, and so the expected number of triangles in $G(n, p)$ is asymptotically $n^{3} p^{3} / 6$. This suggests the critical range $p=\Theta(1 / n)$. Indeed, Erdös and Rényi proved that if $p=p(n) \ll 1 / n$ then $\lim _{n \rightarrow \infty} \operatorname{Pr}[G(n, p) \vDash A]=0$, while if $p=p(n) \gg$ $1 / n$ then $\lim _{n \rightarrow \infty} \operatorname{Pr}[G(n, p) \models A]=1$. (Notation: $f(n) \ll g(n)$ means $\lim _{n \rightarrow \infty} f(n) / g(n)=0$ while $f(n) \gg g(n)$ means $\lim _{n \rightarrow \infty} f(n) / g(n)=+\infty$.) They called $p(n)=1 / n$ a threshold function for this property $A$. As other examples, connectivity has threshold function $(\log n) / n$, containing a clique on four points has threshold function $n^{-2 / 3}$, containing an edge has (easily!) threshold function $n^{-2}$, and every vertex lying in a triangle has threshold function $(\log n)^{1 / 3} n^{-2 / 3}$. It was the observation that threshold functions seemed to be of the form $(\log n)^{\alpha} n^{-\beta}$ with $\alpha, \beta$ rational that motivated our current line of research.

What can we say about the possible threshold functions of properties $A$ ? Not much if we place no restrictions on $A$. For example, the property that the number of edges is even shows no threshold function behavior. If we restrict

Received by the editors May 21, 1990 and, in revised form, February 15, 1991.

1991 Mathematics Subject Classification. Primary 05C80. 
$A$ to be monotone, Bollobás and Andrew Thomason [BT] have general results. Throughout this paper we restrict ourselves to the properties $A$ expressible in the first order theory of graphs, as described in $\S 2$. This restriction is somewhat artifical to graph theorists (connectivity, for example, is not a first order property) though natural to logicians.

Ron Fagin [F2] and, independently, Glebskii, Kogan, Liogonkii, and Talanov [GKLT] proved that for $p=1 / 2$ and any first order $A$

$$
\lim _{n \rightarrow \infty} \operatorname{Pr}[G(n, p) \models A]=0 \text { or } 1 \text {. }
$$

In $G(n, 1 / 2)$ all graphs have equal probability. (We always count labelled graphs, not isomorphism types.) These authors used enumerative rather than probabilistic language: for any first order $A$ the proportion of graphs on $[n]$ satisfying $A$ approaches either zero or one. We shall say that a function $p=$ $p(n)$ satisfies the Zero-One Law if for all statements $A$ of the first order theory of graphs

$$
\lim _{n \rightarrow \infty} \operatorname{Pr}[G(n, p) \models A]=0 \text { or } 1 .
$$

In this paper we give a nearly complete characterization of those $p=p(n)$. Roughly speaking, these $p=p(n)$ lie in the cracks between the threshold functions. At the threshold function $\operatorname{Pr}[G(n, p) \models A]$ is moving from zero to one. For example, when $A$ is the property of containing a triangle and $p=c / n$ one can show $\lim _{n \rightarrow \infty} \operatorname{Pr}[G(n, p) \vDash A]=1-e^{-c^{3} / 6}$ so that $p=c / n$ does not satisfy the Zero-One Law. The $p=p(n)$ satisfying the Zero-One Law are the dull $p(n)$ where "nothing happens" as opposed to the interesting $p(n)$, which are the threshold functions.

Saharon Shelah and the second author [SS] showed that if $0<\alpha<1$ and $\alpha$ is irrational then $p=n^{-\alpha}$ satisfies the Zero-One Law. This current work may be regarded as a sequel to [SS], though much of the argument is independent.

In their 1960 paper Erdös and Rényi [ER] completely analyzed the threshold function for containing a fixed graph $H$. When $H$ has $v$ vertices and $e$ edges the expected number of copies of $H$ in $G(n, p)$ is $\Theta\left(n^{v} p^{e}\right)$, which suggests $p(n)=n^{-v / e}$ to be the threshold function. Erdös and Rényi showed this was indeed the case provided $H$ had no subgraph $H^{\prime}$ with $v^{\prime}$ vertices, $e^{\prime}$ edges, and $e^{\prime} / v^{\prime}>e / v$. (This is a natural condition since the threshold function for containing $H$ must be at least as large as the threshold function for containing any subgraph $H^{\prime}$.) For each integer $k \geq 1, n^{-(k+1) / k}$ is the threshold function for having a path of length $k$. A triangle shows, as previously discussed, $n^{-1}$ to be a threshold function. For any rational $\alpha=a / b$ with $0<\alpha<1, n^{-\alpha}$ is the threshold function for some particular graph. Let $S$ consist of $1,(k+1) / k$ for $k=1,2, \ldots$ and all rationals $\alpha \in(0,1)$. For each $\alpha \in S$ there is a graph $H_{\alpha}$ so that $n^{-\alpha}$ is the threshold function for the existence of a copy of $H_{\alpha}$ in $G$. Furthermore, when $p=c n^{-\alpha}$ the probability of $G(n, p)$ containing a copy of $H_{\alpha}$ approaches a limit strictly between zero and one. For $p=p(n)$ to satisfy the Zero-One Law we must have $p \ll n^{-\alpha}$ or $p \gg n^{-\alpha}$ for all $\alpha \in S$. 
In [SS] it is shown that if $p \ll n^{-2}$ or $n^{-(k+1) / k} \ll p \ll n^{-(k+2) /(k+1)}$ for some $k=1,2, \ldots$ then $p=p(n)$ does satisfy the Zero-One Law. Call $p=p(n)$ very sparse if it does not satisfy $p>n^{-1+o(1)}$, i.e., if there is an $\varepsilon>0$ so that $p(n)<n^{-1-\varepsilon}$ for infinitely many $n$. Suppose such $p=p(n)$ satisfies the Zero-One Law. Take $k>1 / \varepsilon$. If $p(n)>n^{-(k+1) / k}$ for infinitely many $n$ then, considering the property of containment of a path of length $k, p(n)$ would not satisfy the Zero-One Law. Hence $p(n) \leq n^{-(k+1) / k}$ for all sufficiently large $n$ so that $p(n) \ll n^{-(k+2) /(k+1)}$. For $1 \leq i \leq k$ we must have either $p(n) \ll$ $n^{-(i+1) / i}$ or $p(n) \gg n^{-(i+1) / i}$. Hence $p(n)$ must fit "between the cracks": either $p(n) \ll n^{-2}$ or $n^{-(i+1) / i} \ll p(n) \ll n^{-(i+2) /(i+1)}$ for some $1 \leq i \leq k$. Thus the conditions of [SS] completely characterize those very sparse $p=p(n)$ that satisfy the Zero-One Law.

Henceforth we assume $p>n^{-1+o(1)}$.

For such $p$ to satisfy the Zero-One Law we must have

$$
p=n^{-\alpha+o(1)}
$$

for some $\alpha$. For otherwise we would have $p=n^{-\beta+o(1)}$ on one subsequence and $p=n^{-\gamma+o(1)}$ on another with $1 \geq \beta>\gamma \geq 0$. There would be a rational $\alpha$ strictly between $\beta$ and $\gamma$ and then the sentence $G(n, p) \supset H_{\alpha}$ would have probabilities approaching zero and one on the respective subsequences. When $\alpha$ is irrational it is shown in [SS] that any $p=n^{-\alpha+o(1)}$ does satisfy the Zero-One Law. The situation with $\alpha=1$ will be treated in $\S 6$. When $\alpha=0$ the classic results of [F2, GKLT] give that if $p>n^{-\varepsilon}$ for all positive $\varepsilon$ and $1-p>n^{-\varepsilon}$ for all positive $\varepsilon$ then the Zero-One Law is satisfied. For $p$ so close to 1 that the second condition is not satisfied we reduce to $p=o(1)$ by noting, interchanging adjacency with nonadjacency, that $p$ satisfies the Zero-One Law if and only if $1-p$ does.

This leaves us with the central object of this paper: $p=n^{-\alpha+o(1)}$ where $\alpha$ is a rational number between zero and one. As $p=n^{-\alpha}$ is itself a threshold function, we split the possible $p$ into two categories:

$$
p \gg n^{-\alpha} \quad \text { and } \quad p=n^{-\alpha+o(1)}
$$

and

$$
p \ll n^{-\alpha} \quad \text { and } \quad p=n^{-\alpha+o(1)} .
$$

Suppose $\alpha=1 / 7$ and consider $p \gg n^{-1 / 7}$. The property that every seven vertices have a common neighbor has threshold function $(\log n)^{1 / 7} n^{-1 / 7}$. We show (Theorem 2) that if $p \gg(\log n)^{1 / 7} n^{-1 / 7}$ but still $p=n^{-1 / 7+o(1)}$, then $p=p(n)$ satisfies the Zero-One Law. Thus, for example, $n^{-1 / 7} \sqrt{\log n}$ cannot be the threshold function of any first order property. More precisely, let $A_{r}$ be the property that every seven vertices have at least $r$ common neighbors. If $\neg A_{r}$ then there exist $x_{1}, \ldots, x_{7}$ and $y_{1}, \ldots, y_{s}$ with $s \leq r$ with all $y_{j}$ adjacent to all $x_{i}$ and no other $z$ adjacent to all $x_{i}$. We bound $\operatorname{Pr}\left[\neg A_{r}\right]$ by 
the expected number of such configurations,

$$
\operatorname{Pr}\left[\neg A_{r}\right] \leq \sum_{s=0}^{r}\left(\begin{array}{l}
n \\
7
\end{array}\right)\left(\begin{array}{l}
n \\
s
\end{array}\right) p^{7 s}\left(1-p^{7}\right)^{n-7-s} .
$$

Some calculation ( $s=r$ being the main term) gives that if

$$
p=[7(\log n+\omega \log \log n) / n]^{1 / 7}
$$

and $\omega=\omega(n) \rightarrow \infty$ then $\operatorname{Pr}\left[\neg A_{r}\right] \rightarrow 0$ for all $r$. We prove in $\S 2$ that these $A_{r}$ give the "final threshold functions" with $p=n^{-1 / 7+o(1)}$. That is, if $p$ is this large but still $p=n^{-1 / 7+o(1)}$ then $p$ satisfies the Zero-One Law. Actually we state our result for any rational $\alpha=a / b \in(0,1)$.

To do this we define in $\S 3$ an explicit axiom system $T$. In $\S 4$ we show that all of the axioms $A$ of $T$ satisfy

$$
\lim _{n \rightarrow \infty} \operatorname{Pr}[G(n, p) \vDash A]=1
$$

for such $p$. This requires some technically difficult probability results on random graphs. In $\S 3$ we show that $T$ is complete. Here there is no probability; this is a completeness result in logic. Of possible independent interest we have defined a countable family of complete theories $T=T^{\alpha}$, one for each rational $\alpha \in(0,1)$. Indeed $T^{\alpha}$, though we do not do so here, may also be defined for any irrational $\alpha \in(0,1)$. These theories are distinct. For let $0<\beta<\gamma<1$ (rational or irrational). Fix $\alpha \in(\beta, \gamma), \alpha$ rational, and let $A$ be a property with threshold function $n^{-\alpha}$. Then $A$ is a theorem of $T^{\beta}$, as $n^{-\beta} \gg n^{-\alpha}$, while $\neg A$ is a theorem of $T^{\gamma}$, as $n^{-\gamma} \ll n^{-\alpha}$.

In [SS] we showed that if $n^{-1 / 7}<p<(\log n)^{1 / 7-\varepsilon} n^{-1 / 7}$ for $\varepsilon>0$ fixed then $p=p(n)$ does not satisfy the Zero-One Law. When $\alpha=a / b$ this holds when $n^{-\alpha}<p<(\log n)^{1 / b-\varepsilon} n^{-\alpha}$. This leaves a small gap in our characterization of $p \gg n^{-\alpha}, p=n^{-\alpha+o(1)}$, which satisfy the Zero-One Law.

In the other direction suppose, again for $\alpha=1 / 7$, that $p \ll n^{-1 / 7}$ and $p=n^{-1 / 7+o(1)}$. We parameterize $p=n^{-1 / 7-1 / \kappa(n)}$ so that $\kappa(n) \rightarrow \infty$ and the slower $\kappa(n)$ grows the smaller $p=p(n)$ is. We show in $\S 5$ that even if $\kappa(n)$ has the growth rate of the inverse Ackermann function, $p=p(n)$ will not satisfy the Zero-One Law. We further show that no recursive function $p=p(n)$ of this type satisfies the Zero-One Law. For example, $n^{-1 / 7} \log ^{-10} n$ and $n^{-1 / 7} e^{-\sqrt{\log n}}$ do not satisfy the Zero-One Law. Yet, by a fairly simple diagonal argument (Theorem 4) we show that there are $p=p(n)$ of this type satisfying the Zero-One Law. This argues that a complete characterization of $p=p(n)$ in this range satisfying the Zero-One Law is not possible.

In [SS] it is shown how to represent fragments of arithmetic in $G(n, p)$ for $p$ near $n^{-1 / 7}$. In particular, the following result is given there. 
Theorem 1. There is a first order $B$ such that for any $p=(q / n)^{1 / 7}$ with

$$
n^{-1 / \log \log \log \log \log n}<q(n)<\frac{\log n}{\log \log \log \log \log n},
$$

$\operatorname{Pr}[G(n, p) \models B]$ does not approach a limit in $n$.

The choice of $\alpha=1 / 7$ and of $\log \log \log \log \log n$ were somewhat arbitrary. Let $f(n)$ be any slow growing function approaching infinity definable in fragments of arithmetic, e.g., the inverse Ackermann function. Let $\alpha=a / b \in$ $(0,1)$, expressed in lowest terms. Then there is a first order $B$ so that for any $p=q^{1 / b} n^{-a / b}$ with

$$
n^{-1 / f(n)}<q<(\log n) / f(n)
$$

again $\operatorname{Pr}[G(n, p) \models B]$ does not approach a limit in $n$. Except for the small gap near $q=\log n$ the recursive $p=n^{-\alpha+o(1)}$ that satisfy the Zero-One Law are those satisfying the growth condition of Theorem 2 .

We say $A$ hold a.s. (almost surely) if $\operatorname{Pr}[G(n, p) \vDash A] \rightarrow 1$.

\section{PASSING $n^{-\alpha}$-PRELIMINARIES}

This part of the paper is devoted to the proof of the following result.

Theorem 2. Let $a, b, a<b$ be relatively prime natural numbers and

$$
p(n)=\left((b-a+1)(\log n+\omega \log \log n) n^{-a}\right)^{1 / b},
$$

where $\omega=\omega(n)$ is a function that tends to infinity slowly enough to have $p(n)=$ $n^{-a / b+o(1)}$.

Then $p(n)$ satisfies the Zero-One Law.

Since the proof of Theorem 2 will follow ideas presented in [SS] and [S1], we shall try also, whenever possible, to employ notation from these papers. Henceforth we shall assume $a, b, a<b$ to be fixed, although arbitrary, relatively prime natural numbers and $\omega(n)$ will denote a function such that $\omega(n) \rightarrow \infty$, but for $p(n)$ defined by $(*)$, we have $p(n)=n^{-a / b+o(1)}$.

Here and below we deal exclusively with the first order theory of graphs. It contains a countable number of variables $x, y, z, \ldots$, brackets, two binary predicates equality $=$ and adjacency $\sim$, negation $\neg$, conjugacy $\wedge$, and existential qualifier $\exists$. Occasionally we shall also use other Boolean connectives $\vee$, $\Rightarrow, \Leftrightarrow$, and universal quantifier $\forall$ all of which, however, can be defined using $\neg, \wedge$, and $\exists$. Variable arguments in the first order graph theory denote always vertices, not subsets, and all formulae are of finite length. Thus, we may state the fact that a graph contains an isolated edge

$$
\exists x \exists y \forall z: x \sim y \wedge x \nsim z \wedge y \nsim z,
$$

but we cannot express the existence of cut edges in a graph.

For a graph $G,|G|$ and $e(G)$ respectively denotes the number of vertices and edges in $G$. Note that we shall use the same letter to denote a graph and its 


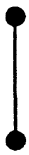

(i)

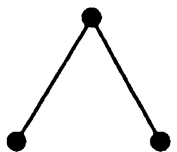

(ii)

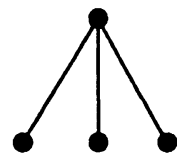

(iii)

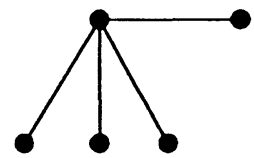

(iv)

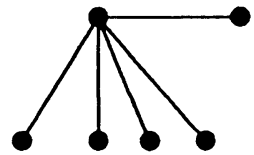

(v)

FIGURE 1

set of vertices. A rooted graph is a pair $(R, H)$, where $H$ is a graph and $R$ is a subset of $H$, vertices of which we shall call roots. Typically, we shall identify the set of vertices of $(R, H)$ with $[h]=\{1,2, \ldots, h]$, where $R=[r]$, and say that $(R, H)$ is of type $(v, e)$ when $v=h-r=|H|-|R|$ and $e$ denotes the number of edges of $H$ with at least one end outside $R$. For a rooted graph $(R, H)$ with a vertex set $[h]$ an $(R, H)$ extension of sequence $\left(x_{1}, x_{2}, \ldots, x_{r}\right)$ of vertices of a graph $G$ is a set $\left\{x_{1}, x_{2}, \ldots, x_{r}, y_{1}, y_{2}, \ldots, y_{h-r}\right\}$ for which there exists a bijection

$$
\sigma:[h] \rightarrow\left\{x_{1}, x_{2}, \ldots, x_{r}, y_{1}, y_{2}, \ldots, y_{h-r}\right\}
$$

such that $\sigma(i)=x_{i}$ for $i=1,2, \ldots, r$ and for every $\{i, j\}$, where $1 \leq i<$ $j \leq h$ and $j>r,\{\sigma(i), \sigma(j)\}$ is an edge of $G$ if $\{i, j\}$ is an edge of $(R, H)$. Let $\operatorname{Ext}(R, H)$ denote the first order property that every $\left(x_{1}, \ldots, x_{r}\right)$ has an $(R, H)$ extension. More precisely, for any particular $(R, H)$ the property $\operatorname{Ext}(R, H)$ is expressible in the first order language.

In the five examples of Figure $1, H$ is the graph pictured and $R$ is the set of vertices at the bottom level. In (i) $\operatorname{Ext}(R, H)$ is the first order property that for all $x_{1}$ there exists $y_{1}$ adjacent to $x_{1}$, i.e., that no vertex is isolated. In (ii) $\operatorname{Ext}(R, H)$ is that every two vertices have a common neighbor and in (iii) that every three vertices have a common neighbor.

We split all rooted graphs into two classes. A rooted graph $(R, H)$ of type $(v, e)$ is sparse if $b v \geq a e$ and dense otherwise. Moreover, a rooted graph $(R, H)$ is safe if $\left(R, H^{\prime}\right)$ is sparse for every subgraph $H^{\prime}$ of $H$ (including $\left.H^{\prime}=H\right)$ and an $(R, H)$ is rigid if $(S, H)$ is dense for each $S$ such that $R \subseteq S \subseteq H$ (including $R=S$ ). Note that safe implies sparse and rigid implies dense.

Examples. Let $a=1, b=2$. Then (i) is safe, (ii) is safe (barely), (iii) is rigid, (iv) sparse but not safe, and (v) dense but not rigid. Let $p=p(n)$ satisfy the condition of Theorem 2. For a given $x_{1}, \ldots, x_{r} \in G(n, p)$ the expected number of $(R, H)$ extensions is $(n-r){ }_{v} p^{e} \sim n^{v} p^{e}$ and this suggests that the threshold function for $\operatorname{Ext}(R, H)$ is near $n^{-v / e}$. Let $p=p(n)$ satisfy the condition of Theorem 2. Then $\operatorname{Ext}(R, H)$ holds a.s. if and only if $(R, H)$ is safe. The if part of this statement (and more) is shown in Lemma 5. For the only if part (which we do not actually use) suppose $(R, H)$ is not safe and let $\left(R, H^{\prime}\right)$ be dense. The expected number of $\left(R, H^{\prime}\right)$ extensions of randomly 
chosen $x_{1}, \ldots, x_{r} \in G(n, p)$ is then $o(1)$ so that almost surely there is no $\left(R, H^{\prime}\right)$, hence no $(R, H)$, extension.

In [SS] a number of simple facts about safe and rigid graphs are shown. We shall use two of them.

Fact 1. If both $(R, S)$ and $(S, H)$ are rigid then so is $(R, H)$.

Proof. For every $U$ such that $R \subseteq U \subseteq H$, rooted graph $(R, U \cap S)$ is dense since $(R, S)$ is rigid and $(S, U \cap(H-S))$ is dense since $(S, H)$ is rigid. Thus $(R, U)$ is dense.

Fact 2. If $(R, H)$ is dense then $\left(R, H^{\prime}\right)$ is rigid for some $H^{\prime}, R \subseteq H^{\prime} \subseteq H$. Proof. Choose minimal $H^{\prime}$ such that $R \subseteq H^{\prime} \subseteq H$ and $\left(H^{\prime}, H\right)$ is sparse (when such $H^{\prime}$ does not exist set $H^{\prime}=H$ ). If $\left(R, H^{\prime}\right)$ is not rigid then for some $U, R \subseteq U \subseteq H^{\prime},(R, U)$ is dense and so $(U, H)$ is sparse contradicting minimality of $H^{\prime}$.

Now we define a notion crucial for our argument. For $t \geq 0$ and sequence $\left(x_{1}, x_{2}, \ldots, x_{r}\right)$ of vertices of graph $G$ the $t$-closure of $\left(x_{1}, x_{2}, \ldots, x_{r}\right)$ in $G$, denoted by $\operatorname{cl}_{t}\left(x_{1}, x_{2}, \ldots, x_{r}\right)$, is a union of all $(R, H)$ extensions of $\left(x_{1}, x_{2}, \ldots, x_{r}\right)$ over all rooted rigid graphs $(R, H)$ with $|H|-|R| \leq t$. When no such extension is possible or $t=0$ then $\mathrm{cl}_{t}\left(x_{1}, x_{2}, \ldots, x_{r}\right)=\left\{x_{1}, x_{2}, \ldots\right.$, $\left.x_{r}\right\}$. Note that for any fixed $t$ the formula $y \in \operatorname{cl}_{t}\left(x_{1}, x_{2}, \ldots, x_{r}\right)$ is a first order predicate as we may list the potential $(R, H)$ extensions.

Example. With $a / b=1 / 2, \mathrm{cl}_{1}\left(x_{1}, x_{2}, x_{3}\right)$ consists of $x_{1}, x_{2}, x_{3}$ plus all common neighbors $y$. In $G(n, p)$ with $p=n^{-1 / 2+o(1)}$ most $x_{1}, x_{2}, x_{3}$ have no common neighbors but some will. Roughly $\mathrm{cl}_{t}\left(x_{1}, \ldots, x_{r}\right)$ gives those $y$ that are "special" with respect to $x_{1}, \ldots, x_{r}$.

Finally, we say that $\left\{x_{1}, x_{2}, \ldots, x_{r}, y_{1}, y_{2}, \ldots, y_{h-r}\right\}$ is a t-generic $(R, H)$ extension of $\left(x_{1}, x_{2}, \ldots, x_{r}\right)$ if it is an $(R, H)$ extension,

$$
y_{i} \notin \mathrm{cl}_{t}\left(x_{1}, x_{2}, \ldots, x_{r}\right) \quad \text { for } i=1,2, \ldots, h-r
$$

and

$$
\begin{aligned}
& \operatorname{cl}_{t}\left(x_{1}, x_{2}, \ldots, x_{r}, y_{1}, y_{2}, \ldots, y_{h-r}\right) \\
& \quad=\operatorname{cl}_{t}\left(x_{1}, x_{2}, \ldots, x_{r}\right) \cup\left\{y_{1}, y_{2}, \ldots, y_{h-r}\right\} .
\end{aligned}
$$

Again note that for any fixed $t$ this is a first order predicate in the variables $x_{1}, x_{2}, \ldots, x_{r}, y_{1}, y_{2}, \ldots, y_{h-r}$.

Example. With $a / b=1 / 2$ and example (ii), a 1-generic $(R, H)$ extension of $\left(x_{1}, x_{2}\right)$ is by a common neighbor $y$ such that $x_{1}, x_{2}, y$ have no common neighbors. With $p=\left(2(\log n+\omega \log \log n) n^{-1}\right)^{1 / 2}, p=n^{-1 / 2+o(1)}$ almost surely every $x_{1}, x_{2}$ have such a $y$. This may not be unexpected as for such $p$ every $x_{1}, x_{2}$ have many common neighbors $y$ and few $x_{1}, x_{2}, y$ have common neighbors $z$. The proof (Lemma 5) is quite technical. 


\section{THE AXIOM SYSTEM-DEFINITION AND THE PROOF OF COMPLETENESS}

In this section we define an axiom system $T$ and show that it is complete in the first order theory of graphs. The definition of $T$ is motivated by consideration of those $A$ that hold a.s. for $p=p(n)$ satisfying the conditions of Theorem 2. The actual definition of $T$ and proof of completeness, however, involve no probability whatsoever.

Let us first define two kinds of statements. For each graph $H$ for which $b|H|<a e(H)$ the nonexistence axiom $A(H)$ says

"there does not exist a copy of $H$,"

whereas for $t \geq 0$ and safe rooted graph $(R, H)$ the generic extension axiom $A(t, R, H)$ is defined as a statement:

"for every sequence $\left\{x_{1}, x_{2}, \ldots, x_{r}\right\}$ there exists a $t$-generic

$(R, H)$ extension of $\left(x_{1}, x_{2}, \ldots, x_{r}\right)$."

Note that both $A(H)$ and $A(t, R, H)$ are statements of first order theory of graphs.

Now define $T$ to be a system containing nonexistence axioms $A(H)$ for all graphs $H$ with $b|H|<a e(H)$ and generic extension axioms $A(t, R, H)$ for all $t$ and safe rooted graphs $(R, H)$. Following customary usage $\vdash_{T} A$ means that $A$ is a theorem of the theory $T$.

Let us start with the simple fact that, in $T$, all $t$-closures are bounded.

Fact 3. For each $r$, $t$ there exists $N=N(r, t)$ such that

$$
\vdash_{T}\left|\operatorname{cl}_{t}\left(x_{1}, x_{2}, \ldots, x_{r}\right)\right| \leq N \text {. }
$$

Proof. Since every rigid extension of type $(v, e)$ satisfies $b v<a e$ and there are only finitely many types of extensions of size smaller than $t$, there exists $\varepsilon>0$ such that for every such extension $b v-a e>\varepsilon$ holds. Choose $K$ such that $r-K \varepsilon<0$ and set $N=r+t K$. If there were a $t$-closure of size larger than $N$ it could be broken into $r$ initial points and then at least $K$ extensions of types $\left(v_{i}, e_{i}\right)$ with all $b v_{i}-a e_{i}<-\varepsilon$; so in total it would contain $v=r+\sum_{i} v_{i}$ vertices and $e=\sum_{i} e_{i}$ edges but

$$
b v-a e \leq r-\varepsilon K<0,
$$

which would violate some of nonexistence axioms. This is provable in $T$ since there are less than $2^{r N^{2}}$ possible extensions of $N$ points and one can prove by examining them one by one that none of them is a $t$-closure of $\left(x_{1}, x_{2}, \ldots, x_{r}\right)$.

Example. With $a / b=1 / 2$,

$$
\vdash_{T}\left|\mathrm{cl}_{1}\left(x_{1}, x_{2}, x_{3}\right)\right| \leq 7 \text {. }
$$

Since the existence of $x_{1}, x_{2}, x_{3}$ and $w_{1}, \ldots, w_{7}$ with all $x_{i}$ adjacent to all $w_{j}$ would violate the nonexistence axiom with $H=K_{3,7}$, the complete bipartite graph with $|H|=10, e(H)=21$. 
Fact 4. For every $r, t^{\prime}$, and $t^{\prime \prime}$ there exists $t=t\left(r, t^{\prime}, t^{\prime \prime}\right)$ such that

$$
\vdash_{T} \mathrm{cl}_{t^{\prime}}\left(\mathrm{cl}_{t^{\prime \prime}}\left(x_{1}, x_{2}, \ldots, x_{r}\right)\right) \subseteq \mathrm{cl}_{t}\left(x_{1}, x_{2}, \ldots, x_{r}\right) .
$$

Proof. It follows immediately from Facts 1 and 3 after setting $t=$ $N\left(N\left(t^{\prime \prime}, r\right), t^{\prime}\right)$.

The next fact is crucial. It essentially states that for large $t$ the knowledge of $\mathrm{cl}_{t}\left(x_{1}, \ldots, x_{r}\right)$ determines the set of possible $\mathrm{cl}_{t^{\prime}}\left(x_{1}, \ldots, x_{r}, y\right), t^{\prime}$ small.

Fact 5. For every $r, t^{\prime}$, and $H^{\prime}$, a possible value of $\mathrm{cl}_{t^{\prime}}\left(x_{1}, x_{2}, \ldots, x_{r}, y\right)$, there is a $t$ such that for every possible value $H$ of $\mathrm{cl}_{t}\left(x_{1}, x_{2}, \ldots, x_{r}\right)$, either

$$
\vdash_{T} \mathrm{cl}_{t}\left(x_{1}, x_{2}, \ldots, x_{r}\right) \cong H \Rightarrow \exists_{y} \mathrm{cl}_{t^{\prime}}\left(x_{1}, x_{2}, \ldots, x_{r}, y\right) \cong H^{\prime}
$$

or

$$
\vdash_{T} \mathrm{cl}_{t}\left(x_{1}, x_{2}, \ldots, x_{r}\right) \cong H \Rightarrow \neg \exists_{y} \mathrm{cl}_{t^{\prime}}\left(x_{1}, x_{2}, \ldots, x_{r}, y\right) \cong H^{\prime} .
$$

Proof. Assume first that $y \in \mathrm{cl}_{\left|H^{\prime}\right|}\left(x_{1}, x_{2}, \ldots, x_{r}\right)$ in $H^{\prime}$. Then from Fact 4, for $t$ large enough

$$
\vdash_{T} \mathrm{cl}_{t^{\prime}}\left(\mathrm{cl}_{\left|H^{\prime}\right|}\left(x_{1}, x_{2}, \ldots, x_{r}\right)\right) \subseteq \mathrm{cl}_{t}\left(x_{1}, x_{2}, \ldots, x_{r}\right) .
$$

Now, since $y \in \operatorname{cl}_{\left|H^{\prime}\right|}\left(x_{1}, x_{2}, \ldots, x_{r}\right) \subseteq \mathrm{cl}_{t}\left(x_{1}, x_{2}, \ldots, x_{r}\right)$, one can examine all possible structures of $\operatorname{cl}_{t}\left(x_{1}, x_{2}, \ldots, x_{r}\right)$ (due to Fact 3 there are finitely many of them) checking if $\mathrm{cl}_{t^{\prime}}\left(x_{1}, x_{2}, \ldots, x_{r}, y\right) \cong H^{\prime}$ for some $y \in$ $\operatorname{cl}_{\left|H^{\prime}\right|}\left(x_{1}, x_{2}, \ldots, x_{r}\right)$.

Now suppose that $y \notin \mathrm{cl}_{\left|H^{\prime}\right|}\left(x_{1}, x_{2}, \ldots, x_{r}\right)$ and set $H$ to be the $\left|H^{\prime}\right|$ closure of $\left(x_{1}, x_{2}, \ldots, x_{r}\right)$ in $H^{\prime}$. Note that rooted graph $\left(H, H^{\prime}\right)$ is safe. Indeed, otherwise for a minimal rigid rooted graph $\left(H, H^{\prime \prime}\right)$, which would exist due to Fact 2, we would have $H^{\prime \prime} \subseteq \mathrm{cl}_{\left|H^{\prime \prime}\right|}\left(x_{1}, x_{2}, \ldots, x_{r}\right)$ contradicting the choice of $H$. Set $t^{\prime}=t$. Then from generic extention axiom $A\left(t, H, H^{\prime}\right)$ we get

$$
\vdash_{T} \exists_{y} \mathrm{cl}_{t^{\prime}}\left(x_{1}, x_{2}, \ldots, x_{r}, y\right) \cong H^{\prime} \Leftrightarrow \operatorname{cl}_{t}\left(x_{1}, x_{2}, \ldots, x_{r}\right) \cong H .
$$

Fact 6. For every formula $P\left(x_{1}, x_{2}, \ldots, x_{r}\right)$ there is a $T$ so that for each possible $H^{\prime}=\mathrm{cl}_{t}\left(x_{1}, x_{2}, \ldots, x_{r}\right)$ either

$$
\vdash_{T} \mathrm{cl}_{t}\left(x_{1}, x_{2}, \ldots, x_{r}\right) \cong H^{\prime} \Rightarrow P
$$

or

$$
\vdash \operatorname{cl}_{t}\left(x_{1}, x_{2}, \ldots, x_{r}\right) \cong H^{\prime} \Rightarrow \neg P .
$$

Proof. The proof goes by the induction on the structure of $P$. For elementary formulae $x=y$ and $x \sim y$ take $t=0$. If it holds for $P$ then it is valid for $\neg P$ with the same $t$. If it holds for $P$ and $Q$ with $t_{P}$ and $t_{Q}$ then it is true for both of them with $t=\max \left(t_{P}, t_{Q}\right)$ and so it holds for $P \wedge Q$ with this $t$. Finally suppose that $P$ is of the form

$$
P: \exists_{y} Q\left(x_{1}, x_{2}, \ldots, x_{r}, y\right) \text {. }
$$


Then by induction it holds for $Q$ for some $t^{\prime}$ so the assertion follows from Fact 5.

Now comes the main result of this section.

Lemma 1. $T$ is a complete set of axioms of the first order theory of graphs.

Proof. Sentences $P$ are formulae with no free variables, so $r=0$. Due to Fact 6 , for some $t, \mathrm{cl}_{t}(\varnothing)$ determines $P$. Nonexistence axioms say however that $\mathrm{cl}_{t}(\varnothing)=\varnothing$ so either $P$ or $\neg P$ is a theorem of $T$.

\section{THE AXIOMS OF $T$ HOLD ALMOST SURELY}

Due to Lemma 1 it is enough to show that all axioms of $T$ hold almost surely for $p(n)$ defined by $(*)$. For nonexistence axioms the answer is given by the well-known result of Erdös and Rényi.

Lemma 2 [ER]. If $p=o\left(n^{-e(H) /|H|}\right)$ then a.s. $G(n, p)$ contains no copies of $H$.

Consequently, for $p(n)$ defined by $(*)$ then the probability that for $G(n, p)$ nonexistence axiom $A(H)$ holds tends to 1 as $n \rightarrow \infty$. The proof that an analogous result holds also for all generic extensions axioms needs a bit more work. Fortunately, the following lemma from [SS] verifies this for a large class of rooted subgraphs $(R, H)$.

Lemma 3 [SS]. Let $(R, H)$ be a rooted subgraph such that all $\left(R, H^{\prime}\right)$ for which $H^{\prime}$ is a subgraph of $H$ are of type $(v, e)$ where $b v<a e$. Then when $p=n^{-b / a+o(1)}$, for every $t$ a.s. $A(t, R, H)$ holds in $G(n, p)$.

Example. $a / b=1 / 2$, example (i), $t=10$. Every $x$ has a neighbor $y$ giving a 10-generic extension. This holds with "lots of room" as there are actually $n^{1 / 2+o(1)}$ neighbors $y$ and almost all of them give a 10 -generic extension.

Let us call a safe rooted graph $(R, H)$ critical if it is of type $(v, e)$ where $b v=a e$. We shall start with the following simple observation.

Fact 7. Let $(R, H)$ be critical, $t \geq 0$ and $p(n)$ be defined by $(*)$. Then there exist a positive constant $\varepsilon=\varepsilon(t)$ and a natural number $N=N(t, R, H)$ such that a.s. for each sequence $\left(x_{1}, x_{2}, \ldots, x_{r}\right)$ of vertices of $G(n, p)$ one of the following possibilities holds: (i) $\left(x_{1}, x_{2}, \ldots, x_{r}\right)$ has no $(R, H)$ extensions; (ii) there exists a t-generic $(R, H)$ extension of $\left(x_{1}, x_{2}, \ldots, x_{r}\right)$; (iii) $\left(x_{1}, x_{2}, \ldots, x_{r}\right)$ is contained in a graph $G$ for which

(a) $|G|<N$,

(b) $b(|G|-r)<a e(G)-\varepsilon$, and

(c) $\left(x_{1}, x_{2}, \ldots, x_{r}\right)$ has no $(R, H)$ extensions $\left\{y_{1}, y_{2}, \ldots, y_{h-r}\right\}$ such that $y \notin G$ for every $j=1,2, \ldots, v$.

Proof. Let $\varepsilon$ be such that $v_{i}^{\prime} b-a e_{i}^{\prime} \leq-\varepsilon$ for all types $\left(v_{i}, e_{i}\right)$ of rigid rooted graphs $\left(S, H^{\prime}\right)$ with $\left|H^{\prime}\right| \leq 2 t+v$ and $N=2 t(b r / \varepsilon+1)+r$. We shall show that for such $\varepsilon$ and $N$ the assertion of Fact 7 remains valid. 
Let $\left(x_{1}, x_{2}, \ldots, x_{r}\right)$ be a sequence of vertices of $G(n, p)$ with at least one $(R, H)$ extensions but with none that are $t$-generic. Take any $(R, H)$ extension of $\left(x_{1}, x_{2}, \ldots, x_{r}\right)$, say $H_{1}^{s}$. Since $H_{1}^{s}$ is not $t$-generic for some rigid graph $\left(S_{1}, H_{1}\right)$ there exists $\left(S_{1}, H_{1}\right)$ extension $H_{1}^{r}$ of some sequence terms of which are taken from $\left\{x_{1}, x_{2}, \ldots, x_{r}\right\} \cup H_{1}^{s}$. Note that graph $G_{1}$ spanned by the set $\left\{x_{1}, x_{2}, \ldots, x_{r}\right\} \cup H_{1}^{s} \cup H_{1}^{r}$ has at most $r+2 t$ vertices and $b|G|-a e(G) \leq b r-\varepsilon$. Now suppose that there exists another $(R, H)$ extension $H_{2}^{s}$ of $\left(x_{1}, x_{2}, \ldots, x_{r}\right)$, disjoint with both $H_{1}^{s}$ and $H_{2}^{r}$. Then, since $H_{2}^{s}$ is not $t$-generic, for some rigid graph $\left(S_{2}, H_{2}\right)$ graph $G(n, p)$ contains $\left(S_{2}, H_{2}\right)$ extension $H_{2}^{r}$ of some sequence from $\left\{x_{1}, x_{2}, \ldots, x_{r}\right\} \cup H_{1}^{s}$. Since $\left(S_{2}, H_{2}\right)$ is rigid, $H_{2}^{r}$ is a rigid extension of $\left\{x_{1}, x_{2}, \ldots, x_{r}\right\} \cup H_{1}^{s} \cup H_{1}^{r} \cup H_{2}^{s}$. (In the extremal case, $H_{2}^{r}$ is a subset of $\left\{x_{1}, x_{2}, \ldots, x_{r}\right\} \cup H_{1}^{s} \cup H_{1}^{r} \cup H_{2}^{s}$ but with some additional edges.) Hence, for a graph $G_{2}=\left\{x_{1}, x_{2}, \ldots, x_{r}\right\} \cup H_{1}^{s} \cup H_{1}^{r} \cup H_{2}^{s} \cup H_{2}^{r}$ we have $\left|G_{2}\right| \leq 4 t+r$ and $b\left|G_{2}\right|-a e\left(G_{2}\right) \leq b r-2 \varepsilon$.

Continue this procedure as long as possible. Clearly it must stop after at most $N$ steps since otherwise we would get a graph $G_{N}$ for which $b\left|G_{N}\right|<a e\left(G_{N}\right)$, which is impossible due to Lemma 2.

We went to show each $A(t, R, H)$ holds a.s. It now suffices to show that a.s. no $\left(x_{1}, \ldots, x_{r}\right)$ satisfies (i) or (ii).

Let us call safe rooted graph $(R, H)$ balanced if there are no critical rooted subgraphs $\left(R, H^{\prime}\right)$ such that $R \subset H^{\prime} \subset H$. The following result follows from a correlation inequality as stated in [S2] (see also [BS] and [JLR]).

Lemma 4. Let $(R, H)$ be a balanced critical rooted graph of type $(v, e)$ and $p(n)$ be given by $(*)$. Then there exists a positive constant $\varepsilon=\varepsilon(a, b,(R, H))>$ 0 such that the probability that a given sequence $\left(x_{1}, x_{2}, \ldots, x_{r}\right)$ of vertices of $G(n, p)$ has no $(R, H)$ extension is less than $\exp \left(-n^{v} p^{e}\left(1-n^{-\varepsilon}\right)\right)$ provided $n$ is large enough.

Let us note the following consequence of Lemma 4.

Fact 8. Let $(R, H), p(n)$ be such as in Lemma 4. Then a.s. each sequence $\left(x_{1}, x_{2}, \ldots, x_{r}\right)$ of vertices of $G(n, p)$ has at least $\omega / 3 b$ pairwise disjoint $(R, H)$ extensions, where two extensions are pairwise disjoint if an intersection of their set of vertices is the set $\left\{x_{1}, x_{2}, \ldots, x_{r}\right\}$ of the roots.

Proof. Suppose that $|H|-|R|=h-r=m a$ and let $X$ denote the number of sequences $\left(x_{1}, x_{2}, \ldots, x_{r}\right)$ of vertices of $G(n, p)$ for which there exists less than $N=\omega / 3 b$ pairwise disjoint $(R, H)$ extensions. Then the expectation of $X$ can be bounded from above by

$$
\begin{aligned}
E X & \leq n^{r} \sum_{i=0}^{N-1}\left[\left(\begin{array}{c}
n \\
m a 2^{h^{2}} p^{m b}
\end{array}\right)\right]^{i} e^{-n^{m a} p^{b m}\left(1-n^{-\varepsilon}\right)} \\
& \leq n^{r}\left[2^{h^{2}} n^{a m} p^{b m}\right]^{N} e^{-n^{a m} p^{b m}\left(1-n^{-\varepsilon}\right)}
\end{aligned}
$$


Now let $m=1$. One can easily see that since $(R, H)$ is balanced, the number of roots of $(R, H)$ cannot be larger than $b-a+1$. Thus

$$
\mathrm{E} X \leq n^{b-a+1} \log ^{2 N} n n^{-(b-a+1)} \log ^{-\omega / b} \rightarrow 0 .
$$

If $m>1$ we may estimate $\mathrm{E} X$ even more crudely,

$$
\mathrm{E} X \leq n^{b-a+1} \log ^{2 N m} n \exp \left(-\log ^{2} n\right) \rightarrow 0 .
$$

Lemma 5. Let $t \geq 0,(R, H)$ be a safe rooted graph and $p(n)$ be defined by (*). Then a.s. generic extension axiom $A(t, R, H)$ holds for $G(n, p)$.

Proof. For each fixed $t$ we shall show Lemma 5 using induction with respect to $|H|-|R|$.

When $|H|-|R|<a$ then $(R, H)$ and each $\left(R, H^{\prime}\right)$, where $H^{\prime} \subset H$ are of the type $\left(v^{\prime}, e^{\prime}\right)$, where $b v^{\prime}<a e^{\prime}$ and the assertion follows from Lemma 3. Thus suppose that the assertion holds for all safe rooted graphs $\left(R^{\prime}, H^{\prime}\right)$ with $\left|H^{\prime}\right|-\left|R^{\prime}\right| \leq s-1$. We shall show that it remains valid also for all safe $(R, H)$ for which $|H|-|R|=s$.

Let us consider two cases:

Case 1. $(R, H)$ is not balanced, i.e., for some $H^{\prime}, R \subset H^{\prime} \subset H,\left(R, H^{\prime}\right)$ is critical.

Then both $\left(R, H^{\prime}\right)$ and $\left(H^{\prime}, H\right)$ are safe, so from the inductional asumption a.s. there is a $t$-generic $\left(R, H^{\prime}\right)$ extension $\left\{x_{1}, x_{2}, \ldots, x_{r}, y_{1}, y_{2}, \ldots, y_{h-r}\right\}$ of each sequence $\left(x_{1}, x_{2}, \ldots, x_{r}\right)$, and, again from the inductional step, each $\left(x_{1}, x_{2}, \ldots, x_{r}, y_{1}, y_{2}, \ldots, y_{h-r}\right)$ has a $t$-generic $\left(H^{\prime}, H\right)$ extension.

Case 2. $(R, H)$ is balanced.

If $(R, H)$ is not critical the assertion follows from Lemma 3, whereas for critical $(R, H)$ it follows from Facts 7 and 8 .

Proof of Theorem 2. It is an immediate consequence of Lemmas 1, 2, and 5.

Remark. It is not hard to see that Theorem 2 is in a way best possible. Namely, for each $a, b, a<b$, there exists a safe rooted graph $(R, H)$ such that $|R|=b-a+1,|H|=a$ (in the case when $a=1, b=7$ it is just a vertex adjacent to seven roots) such that for every constant $C$ and $p(n)=$ $\left((b-a+1)(\log n+C \log \log n) n^{-a}\right)^{1 / b}$ there is a function $p^{\prime}(n)>p(n)$ such that $p^{\prime}(n)=n^{-a / b+o(1)}$ and the probability that some sequence $\left\{x_{1}, \ldots, x_{b-a+1}\right\}$ has exactly $\lceil b C\rceil$ vertex disjoint $(R, H)$ extensions in $G(n, p)$ tends to some constant $D=D(C)$ as $n \rightarrow \infty$, where $0<D<1$. Thus Theorem 2 gives the minimal condition so that if $p=n^{-a / b+o(1)}$ and $p$ is at least that large then $p$ must satisfy the Zero-One Law. The gap in the characterization is that there may be smaller $p$ also satisfying the Zero-One Law.

\section{APPROACHING $n^{-\alpha}$}

Here we ask: what $p=p(n)$ with $p=n^{-\alpha+o(1)}$ and $p \ll n^{-\alpha}$ satisfy the Zero-One Law? We shall show that the restrictions on such $p$ are very severe. 
We shall also show that there are $p$ with that property. While we consider only the exponent $-1 / 7$, the results may be extended to any rational exponent $\alpha \in(0,1)$. We write

$$
p(n)=n^{-1 / 7-1 / \kappa(n)}
$$

and assume $\kappa(n) \rightarrow \infty$. We make heavy use of the results of [SS, §3]. In particular, we may assume

$$
\kappa(n)<\log \log \log \log \log (n),
$$

as otherwise we know $p$ does not satisfy the Zero-One Law. Let $N\left(x_{1}, \ldots, x_{7}\right)$ denote the set of neighbors of $x_{1}, \ldots, x_{7}$. Let $l=l(n)=\lfloor\kappa(n)\rfloor$. The following hold a.s. in $G(n, p)$.

- For every $0 \leq i \leq l-4$ there exist $x_{1}, \ldots, x_{7}$ with precisely $i$ neighbors.

- $l-4 \leq \max \left|N\left(x_{1}, \ldots, x_{7}\right)\right| \leq l+4$.

- For every set $S$ of size at most $10 \mathrm{l}$ and every 6-graph $\mathscr{H}$ on $S$ with at most $l / 10$ hyperedges there is a $w \notin S$ so that for all $v_{1}, \ldots, v_{6} \in S$,

$$
\left\{v_{1}, \ldots, v_{6}\right\} \in \mathscr{H} \Leftrightarrow N\left(v_{1}, \ldots, v_{6}, w\right) \neq \varnothing .
$$

Here we have employed hypergraph terminology: a 6-graph $\mathscr{H}$ on $S$ is a family of subsets of $S$, all of size six, and a hyperedge is an element of $\mathscr{H}$. A 2-graph is a family of subsets, all of size two, which corresponds to the usual notion of graph. Extending and limiting the third property: for every 2-graph $H$ on $S$ of size at most $5 l$ with at most $50 l$ edges there exist $v_{3}, v_{4}, v_{5}, v_{6}, w_{1}, \ldots, w_{500}$ so that for all $v_{1}, v_{2} \in S$

$$
\left\{v_{1}, v_{2}\right\} \in H \Leftrightarrow \bigvee_{i=1}^{500} N\left(v_{1}, \ldots, v_{6}, w_{i}\right) \neq \varnothing
$$

In the first order language let $\operatorname{BIGGER}\left(S, S^{\prime}\right)$ be that for some $v_{3}, v_{4}, v_{5}, v_{6}$ and some $w_{1}, \ldots, w_{500}$ the $H$ defined on $S \cup S^{\prime}$ gives an injection from $S-S^{\prime}$ to $S^{\prime}-S$. In $G(n, p)$ a.s. for every $S, S^{\prime}$ with $\left|S \cup S^{\prime}\right| \leq 5 l \operatorname{BIGGER}\left(S, S^{\prime}\right)$ has the same truth value as the (not first order) statement $\left|S^{\prime}\right| \leq|S|$. We write $\operatorname{MAX}\left(x_{1}, \ldots, x_{7}\right)$ if for all $x_{1}^{\prime}, \ldots, x_{7}^{\prime}$

$$
\operatorname{BIGGER}\left(N\left(x_{1}, \ldots, x_{7}\right), N\left(x_{1}^{\prime}, \ldots, x_{7}^{\prime}\right)\right) \text {. }
$$

As a.s. all $\left|N\left(x_{1}, \ldots, x_{7}\right)\right|<2 l$, a.s. for every $x_{1}, \ldots, x_{7} \operatorname{MAX}\left(x_{1}, \ldots, x_{7}\right)$ has the same truth value as the (not first order) statement that $x_{1}, \ldots, x_{7}$ have the maximum number of common neighbors over all sets of seven vertices. We may say $S$ has size $i(\bmod 10)$, that there is a graph $H$ on $S$ that is the union of 10-cliques plus $i$ more points. For $0 \leq i<10$ let $A_{i}$ be the sentence that there exist $x_{1}, \ldots, x_{7}$ for which $\operatorname{MAX}\left(x_{1}, \ldots, x_{7}\right)$ and so that $N\left(x_{1}, \ldots, x_{7}\right)$ has size that is $i(\bmod 10)$. Then $A_{0} \vee \cdots \vee A_{9}$ holds a.s. so if $p$ satisfies the Zero-One Law precisely one $A_{i}$ holds a.s. This implies there must be a $k^{\prime}(n)$ with $l-4 \leq k^{\prime}(n) \leq l+4$ so that

$$
\max \left|N\left(x_{1}, \ldots, x_{7}\right)\right|=k^{\prime}(n)
$$


a.s. Now set

$$
k(n)=\left\lfloor\left(k^{\prime}(n)\right)^{1 / 3}\right\rfloor .
$$

We may say that a set $S=N\left(x_{1}, \ldots, x_{7}\right)$ has size $k(n)$ : it has maximal size so that there exist $S_{1}, S_{2}$ of the same size, all disjoint, and an injection from $S \times S_{1} \times S_{2}$ into a set $T$ of size $k^{\prime}(n)$. Now $k^{3}(n) \leq k^{\prime}(n) \leq l+$ $4 \leq 50 \mathrm{l}$. Any 3-graph $H$ on $S$ has less than $50 l$ hyperedges so there exist $v_{4}, v_{5}, v_{6}, w_{1}, \ldots, w_{500}$ so that for all $v_{1}, v_{2}, v_{3} \in S$,

$$
\left\{v_{1}, v_{2}, v_{3}\right\} \in H \Leftrightarrow \bigvee_{i=1}^{500} N\left(v_{1}, v_{2}, v_{3}, v_{4}, v_{5}, v_{6}, w_{i}\right) \neq \varnothing
$$

Now let $K$ denote the set of values $k(n)$. A function $p=p(n)$ satisfying the Zero-One Law will determine the set $K$, up to the finite segment. Now let $\mathscr{T}$ be any second order sentence with quantification over unary, binary and ternary predicates as well as normal first order quantification. Set $S=\operatorname{Spec}(\mathscr{T})$, i.e., the set of $m$ for which there is a model of $\mathscr{T}$ containing exactly $m$ elements.

Fact 9. If $p=p(n)$ satisfies the Zero-One Law then for any such $S$ either $K \cap S$ or $K \cap \bar{S}$ must be finite.

Proof. Say $\mathscr{T}$ has ternary predicates $R_{1}, R_{2}, \ldots$ In the theory of graphs make a sentence $A$ that there exist $x_{1}, \ldots, x_{7}$ so that $S=N\left(x_{1}, \ldots, x_{7}\right)$ has size $k(n)$ and on $S$ we have a model of $\mathscr{T}$. We do this by replacing each second order quantified ternary $\exists R_{i}$ by $\exists v_{4}, v_{5}, v_{6}, w_{1}, \ldots, w_{500}$ and replacing $R_{i}\left(v_{1}, v_{2}, v_{3}\right)$ by $\bigvee_{i=1}^{500} N\left(v_{1}, v_{2}, v_{3}, v_{4}, v_{5}, v_{6}, w_{i}\right) \neq \varnothing$. Then a.s. $A$ holds if and only if $k(n) \in S$. (Strictly speaking these ternary relations would be symmetric and hold for three unequal arguments. The somewhat technical modification to handle quantification over all ternary relations is discussed in [SS]. Binary and unary relations are handled similarly. Of course, the symbols used for $v_{4}, \ldots, w_{500}$ must be different in each replacement.) With $p=p(n)$ satisfying the Zero-One Law we must have $k(n) \in S$ being either true for all sufficiently large $n$ or false for all sufficiently large $n$ and as $k(n) \rightarrow \infty$ this implies Fact 9.

This fact gives a great strengthening of the results of [SS]. For example, let $\beta<\varepsilon_{0}$ and let $f_{\beta}$ denote the $\beta$ th function in the transfinite Ackermann heirarchy.

Fact 10. If $\kappa(n)>f_{\beta}^{-1}(n)$ for all sufficiently large $n$ then $p=p(n)$ does not satisfy the Zero-One Law.

Proof. As $k(n) \sim \kappa(n)^{1 / 3}$ this would imply $k(n)>f_{\beta+1}^{-1}(n)$. For $k \in K$ let $k^{+}$denote the next element of $K$ in ascending order. Say $k=k(n)$. Then $k\left[f_{\beta+1}(n)\right]>k$ and

$$
k^{+} \leq k\left[f_{\beta+1}(n)\right] \leq f_{\beta+1}(n) \leq f_{\beta+1}\left(f_{\beta+1}(k)\right) \leq f_{\beta+2}(k) .
$$


But with ternary predicates we may simulate arithmetic and the set $S$ of those $k$ with $f_{\beta+2}^{-1}(k)$ even is a spectrum. Since in $K, k^{+}$is so "near" $k$ it cannot "jump over" the interval $\left[f_{\beta+2}(s), f_{\beta+2}(s+1)\right)$ and so both $S \cap K$ and $\bar{S} \cap K$ would be infinite.

We are indebted to A. Blass (Ann Arbor) and M. Sipser (Cambridge) for the next result, whose proof employs the "delayed diagonalization" technique.

Fact 11. No recursive infinite set $K$ has the property of Fact 9 that either $K \cap S$ or $K \cap \bar{S}$ is finite for every $S=\operatorname{Spec}(\mathscr{T})$.

Proof. Classic results of Fagin [F1] give that every set $S$ of those $n$ accepted by a polynomial time algorithm (and even much more) are of this form. We suppose a recursive $K$ exists and derive a contradiction by considering the following (linear time!) algorithm for a set $S$. Given $n$ set the "clock" to $n$ and check the integers $1,2,3, \ldots$ for membership in $K$. Let $x$ be the largest integer for which $x \in K$ has been determined when the clock runs out. If no such $x$ has been found then accept $n \in S$. Now recursively check if $x \in S$. If $x \in S$ then say $n \notin S$. If $x \notin S$ then say $n \in S$.

Suppose $K \cap \bar{S}$ is finite so there exists $a_{0} \in K$ so that all $x \geq a_{0}$ with $x \in K$ have $x \in S$. Let $n_{0}$ be such that with the clock set at $n \geq n_{0}$ the condition $a_{0} \in K$ is checked. Let $n>n_{0}$ with $n \in K$. With input $n$, the final $x$ for which $x \in K$ is determined has $x \geq a_{0}$, hence $x \in S$. Hence $n \notin S$, a contradiction. If $K \cap S$ is finite a similar contradiction is reached.

Theorem 3. There is no recursive function $p=p(n)$ with $p<n^{-1 / 7}$ and $p=$ $n^{-1 / 7+o(1)}$ satisfying the Zero-One Law.

Proof. If $p(n)$ were recursive then $K$ would be recursive, contradicting Facts 9, 11.

Our next result provides a sharp contrast.

Theorem 4. There exists a function $p=p(n)$ with $p<n^{-1 / 7}$ and $p=n^{-1 / 7+o(1)}$ satisfying the Zero-One Law.

Proof. Order the sentences in the first order theory of graphs $A_{1}, A_{2}, \ldots$ Set

$$
\alpha_{i}=\frac{1}{7}+\frac{\sqrt{2}}{i}
$$

or any sequence of irrational numbers decreasing to $1 / 7$. Set $E_{0}=$ $\left\{\alpha_{1}, \alpha_{2}, \ldots\right\}$. By induction on $i$ we define $a_{1}, \ldots, a_{i}$ and sets $E_{0} \supset E_{1} \supset$ $\cdots \supset E_{i}$, all infinite. With $E_{i-1}$ having been defined split $a \in E_{i-1}$ into two classes according to whether $A_{i}$ or $\neg A_{i}$ holds a.s. with $p=n^{-a}$. As all $a$ are irrational this gives a strict dichotomy. Let $E_{i}$ be the infinite class, or either class if both are infinite. (This step is nonrecursive. Even for $i=1$ there is no decision procedure that determines if $A$ holds a.s. with $p=n^{-a}$ for infinitely many $a \in E_{0}$.) For notational convenience let $B_{i}$ denote either $A_{i}$ or $\neg A_{i}$, whichever gave the class $E_{i}$. Select $a_{i} \in E_{i}, a_{i}<a_{i-1}$, arbitrarily. Note that 
as the $E_{i}$ are a descending sequence we have that for all $i \leq j$ that $B_{i}$ holds a.s. in $G\left(n, n^{-a_{j}}\right)$. For each $j$ we may therefore pick an $n_{j}$ so that for $n \geq n_{j}$

$$
\operatorname{Pr}\left[G\left(n, n^{-a_{j}}\right) \models B_{i}\right] \geq 1-1 / j, \quad 1 \leq i \leq j .
$$

Replacing $n_{j}$ by $\max \left(n_{1}, \ldots, n_{j}\right)$ we can further assure $n_{1} \leq n_{2} \leq \cdots$. Now we define $p=p(n)$ by letting $p(n)$ be arbitrary for $n<n_{1}$ and setting

$$
p(n)=n^{-a_{j}}, \quad n_{j} \leq n<n_{j+1} .
$$

As $a_{j} \rightarrow 1 / 7, p(n)=n^{-1 / 7+o(1)}$. As all $a_{j}>1 / 7, p(n)<n^{-1 / 7}$. For each $i$, we have that for each $j \geq i, \operatorname{Pr}\left[B_{i}\right] \geq 1-1 / j$ for $n_{j} \leq n$ and so $B_{i}$ holds a.s. and therefore $A_{i}$ holds with probability approaching either zero or one.

$$
\text { 6. } p=n^{-1+o(1)}
$$

In this section we assume $p=n^{-1+o(1)}$ throughout and we characterize those $p$ that satisfy the Zero-One Law. In [SS] it is shown that if

$$
\begin{gathered}
p \ll n^{-1}, \\
n^{-1} \ll p \ll n^{-1}(\log n),
\end{gathered}
$$

or

$$
n^{-1}(\log n) \ll p
$$

then $p$ does satisfy the Zero-One Law. When $p=c / n$ the probability that $G=G(n, p)$ is trianglefree approaches $e^{-c^{3} / 6}$. Hence for $p$ to satisfy the Zero-One Law we must have $p \ll n^{-1}$ or $p \gg n^{-1}$. When $p=\log n / n+c / n$ the probability that $G=G(n, p)$ has no isolated points is $e^{-e^{-c}}$. (This is better known as the threshold function for connectivity.) However, in the range $p=\Theta(\log n / n)$ the threshold functions are "tighter" and there is still room for $p$ to satisfy the Zero-One Law. The crucial sentences (for which we gratefully acknowledge the assistance of $\mathrm{N}$. Pippenger) are the following, defined for $k \geq$ $1, s \geq 0$ :

- $A_{k, s}$ : There exist $x_{1}, \ldots, x_{k}$ forming a path of length $k-1, x_{1}$ only adjacent to $x_{2}, x_{i}$ only adjacent to $x_{i-1}, x_{i+1}$ for $1<i<k$, and $x_{k}$ adjacent only to $x_{k-1}$ and precisely $s$ other vertices $y_{1}, \ldots, y_{s}$.

The special case $k=1$ simplifies to

- $A_{1, s}$ : There is a vertex of degree precisely $s$.

Set

$$
p=p_{k, s}(n)=\frac{\log n}{k n}+\frac{(k+s-1) \log \log n}{k n}+\frac{c}{k n} .
$$

There are $\sim n^{k+s} / s$ ! potential $x_{1}, \ldots, x_{k}, y_{1}, \ldots, y_{s}$ and each satisfies the condition with probability $\sim p^{k+s-1}(1-p)^{k n}$. With this $p$ the expected number of such sets is then $e^{-c} / s$ ! and

$$
\operatorname{Pr}\left[A_{k, s}\right] \rightarrow 1-e^{-e^{-c} / s !}
$$


For notational convenience write $p(n)<^{*} q(n)$ if $n(q(n)-p(n)) \rightarrow \infty$ and $p(n)>^{*} q(n)$ if $n(q(n)-p(n)) \rightarrow-\infty$.

Theorem 5. $p=n^{-1+o(1)}$ satisfies the Zero-One Law if and only if

$$
p \ll n^{-1} \quad \text { or } \quad p \gg n^{-1}
$$

and for all $k \geq 1, s \geq 0$

$$
p<^{*} p_{k, s} \text { or } p>^{*} p_{k, s}
$$

The other cases having been handled in [SS] we may assume $p=\Theta(\log n / n)$. In [SS] it has been noticed that for every $m$ a.s. there do not exist $m$ vertices with $m+1$ (or more) edges. For every $m \geq 3, r$ there a.s. do exist (at least) $r$ cycles of size precisely $m$. For every $m, s$ a.s. every $m$ vertices that have $m$ edges have each vertex of degree (at least) $s$. Thus countable models of $G(n, p)$ would consist only of trees and unicyclic components. The unicyclic components are determined: for each $m \geq 3$ there will be countably many components with a single cycle of length $m$ and all degrees infinite. The distinctions come in the tree components.

Suppose $p<^{*} p_{k+1,0}$. For all $m, r$ a.s. there do not exist $m$ vertices joined in a tree containing $k+1$ vertices of degree at most $r$. In the countable models no tree can contain $k+1$ (or more) vertices of finite degree.

Suppose $p>^{*} p_{k-1, s}$ for all $s$. Fix an arbitrary tree $T$ with any $k-1$ specified vertices $v_{1}, \ldots, v_{k-1}$ and with any specified integers $d_{1}, \ldots, d_{k-1}$ with each $d_{i}$ at least the degree of its respective $v_{i}$ in $T$. Then a.s. there will be contained in $G(n, p)$ an induced copy of $T$ with the vertex corresponding to $v_{i}$ having degree precisely $d_{i}$. In the countable models there will be trees having $k-1$ (or less) vertices of finite degree forming all possible finite subtrees.

When $p>^{*} p_{k-1, s}$ for all $s$, and $p<{ }^{*} p_{k+1,0}$ the countable models of $G(n, p)$ are distinguished by the trees containing precisely $k$ vertices of finite degree. Let $T$ be a finite tree on, say, $m$ vertices with distinguished vertices $y_{1}, \ldots, y_{k}$. Suppose further that the $y_{i}$ include all the leaves of $T$. Let $l_{1}, \ldots, l_{k}$ denote the degrees of $y_{1}, \ldots, y_{k}$ respectively in $T$. Let $d_{1}, \ldots, d_{k} \geq 0$. Let $A$ be the event that $G(n, p)$ contains an induced copy of $T$ and that $y_{i}$ has degree precisely $l_{i}+d_{i}$. For this $A$ we set $s=m+d_{1}+\cdots+d_{k}-k \geq 0$ and

$$
p_{A}=\frac{\log n}{k n}+\frac{(k+s-1) \log \log n}{k n} .
$$

Then if $p<{ }^{*} p_{A}, \neg A$ holds a.s. while if $p>^{*} p_{A}$ then $A$ holds a.s.

Suppose $p_{k, s-1}<{ }^{*} p<^{*} p_{k, s}$. Then the countable model of $G(n, p)$ is determined: those tree components with precisely $k$ points of finite degree exist if and only if the points and their degrees match the criteria above. Conversely, for each such $T, y_{1}, \ldots, y_{k}, d_{1}, \ldots, d_{k}$ meeting the criteria there will be countably many such components. 


\section{REFERENCES}

[B] B. Bollobás, Random graphs, Academic Press, New York, 1985.

[BS] R. Boppana and J. Spencer, A useful elementary correlation inequality, J. Combin. Theory Ser. A 50 (1989), 305-307.

[BT] B. Bollobás and A.G. Thomason, Threshold functions, Combinatorica 7 (1986), 35-38.

[ER] P. Erdős and A. Rényi, On the evolution of random graphs, Magyar Tud. Akad. Mat. Kutató Int. Közl 5 (1960), 17-61.

[F1] R. Fagin, Generalized first order spectra and polynomial time recognizable sets, Complexity of Computation (SIAM-AMS Proc., New York, April 18-19, 1973), vol. 7 (R. M. Karp, ed.), 1974, pp. 43-73.

[F2] _ Probabilities on finite models, J. Symbolic Logic 41 (1976), 50-58.

[GKLT] Y. V. Glebskii, D. I. Kogan, M. I. Liogonkii, and Talanov, Range and degree of realizability of formulas in the restricted predicate calculus, Cybernetics 5 (1969), 142-154.

[JLR] S. Janson, T. Luczak, and A. Ruciński, An exponential bound for the probability of nonexistence of a specified subgraph in a random graph (to appear).

[S1] J. Spencer, Countable sparse random graphs, Random Structures and Algorithms 1 (1990), 205-214.

[S2] _ Threshold functions for extension statements, J. Combin. Theory Ser. A 53 (1990), 286-305.

[SS] J. Spencer and S. Shelah, Zero-one laws for sparse random graphs, J. Amer. Math. Soc. 1 (1988), 97-115.

Institute of Mathematics at Adam Mickiewicz University, Matejki 48-49, $60-769$ PozNAN, POLAND

New York University, Courant Institute of Mathematical Sciences, 251 Mercer Street, New York, New York 10012 